\title{
The diameter of the set of boundary slopes of a knot
}

\author{
BEN KLAFF \\ Peter B Shalen
}

\begin{abstract}
Let $K$ be a tame knot with irreducible exterior $M(K)$ in a closed, connected, orientable 3-manifold $\Sigma$ such that $\pi_{1}(\Sigma)$ is cyclic. If $\infty$ is not a strict boundary slope, then the diameter of the set of strict boundary slopes of $K$, denoted $d_{K}$, is a numerical invariant of $K$. We show that either (i) $d_{K} \geq 2$ or (ii) $K$ is a generalized iterated torus knot. The proof combines results from Culler and Shalen [3] with a result about the effect of cabling on boundary slopes.
\end{abstract}

57M15, 57M25; 57M50

\section{Introduction}

Let $K$ be a (tame) knot in a connected, closed, orientable 3-manifold $\Sigma$, such that the exterior $M(K)$ of $K$ is irreducible and $M(K)$ contains no strict essential surface with meridian boundary slope. The diameter $d_{K}$ of the set of all boundary slopes of strict essential surfaces in $M(K)$ is a natural invariant of $K$. (The definition of $d(K)$ and of other specialized terms used here will be reviewed below.) Properties of $d_{K}$ have topological and algebraic meaning for the knot $K$. For example, the main result of Culler and Shalen [2], which implies Neuwirth's conjecture [10] that classical knot groups are nontrivial amalgamated free products, is equivalent to the assertion that under suitable mild restrictions on $K$, we have $d_{K} \neq 0$. (For further discussion of this connection, see Shalen [11].)

It was shown in Culler and Shalen [3] that if $\pi_{1}(\Sigma)$ is trivial and the knot $K$ is nontrivial, then $d_{K} \geq 2$. In this paper we extend this result to the case in which $\pi_{1}(\Sigma)$ is cyclic. Theorem B below asserts that in this situation we still have $d_{K} \geq 2$, unless the knot $K$ belongs to a certain special class of knots that we call generalized iterated torus knots.

When $K$ belongs to the special class just mentioned, then a great deal is known about $K$ and also $\Sigma$ : it is not hard to classify all generalized iterated torus knots, to show 
that a manifold containing such a knot must be a lens space, and to calculate $d_{K}$ when $K$ is such a knot. In many (but not all) cases, it turns out that $d_{K}<2$.

Theorem B is proved by combining one of the results of [3] with another new result, Theorem A, which asserts that a $q$-strand cabling of a knot increases the invariant $d_{K}$ by a factor of at least $q^{2}$.

By combining Theorem $\mathrm{A}$ and Theorem $\mathrm{B}$ we obtain Corollary $\mathrm{C}$ which gives a stronger version of Theorem B for the case of a cable knot. For example, it follows from Corollary C that if $K$ is a cable of a hyperbolic knot in $S^{3}$ then $d_{K} \geq 8$.

Corollary C will be used in Klaff [9], where it is shown that if $\pi_{1}(\Sigma)$ is a finite cyclic group of odd order, and if the knot $K \subset \Sigma$ is not a generalized iterated torus knot, then $d_{K}$ is strictly greater than 2 .

One of the ingredients in the proof of Theorem B is Proposition 1.3, which provides a criterion for deciding whether a surface in a cabled knot exterior-or more generally, in an irreducible, orientable 3-manifold that contains an essential torus-is strict and essential. This result is of independent interest, and is applied by Culler and Shalen [4] in a rather different context.

Before giving formal statements of Theorems A and B, we shall set up some general conventions and define some of the terms we used above.

We shall work in the piecewise linear category throughout this paper.

0.1 If $T$ is a 2-dimensional torus, we define a slope on $T$ to be an isotopy class of homotopically nontrivial simple closed curves in $T$. The set of all slopes on $T$ will be denoted by $\mathcal{S}(T)$.

The isotopy classes of homotopically nontrivial oriented simple closed curves in $T$ are in natural bijective correspondence with elements of $H_{1}(T ; \mathbb{Z})$ which are primitive in the sense of not being divisible by any integer greater than 1 . Thus there is a natural two-to-one map from the set of primitive elements of $H_{1}(T ; \mathbb{Z})$ onto $\mathcal{S}(T)$. We shall denote this map by $\alpha \mapsto\langle\alpha\rangle$. We have $\langle\alpha\rangle=\left\langle\alpha^{\prime}\right\rangle$ if and only if $\alpha^{\prime}= \pm \alpha$.

0.2 If $C$ is a nonempty closed 1 -manifold in a 2-torus $T$, and $C$ has no homotopically trivial components, then all components of $C$ have the same slope $\sigma \in \mathcal{S}(T)$. We call $\sigma$ the slope of $C$.

0.3 A 3-manifold $M$ is irreducible if $M$ is connected and every 2-sphere in $M$ bounds a ball.

An essential surface in an irreducible, orientable 3-manifold $M$ is a two-sided properly embedded surface in $M$ which is nonempty and $\pi_{1}$-injective, and has no 2 -sphere components and no boundary-parallel components. 
0.4 Suppose that $M$ is a compact, orientable irreducible 3-manifold whose boundary components are tori. A connected essential surface in $M$ is called a semifiber if either $F$ is a fiber in a fibration of $M$ over $S^{1}$, or $F$ is the common frontier of two 3-dimensional submanifolds of $M$, each of which is a twisted [0,1]-bundle with associated $\{0,1\}$-bundle $F$. An essential surface $F \subset M$ is termed strict if no component of $F$ is a semifiber. A strict essential surface has no disk components, since an irreducible knot manifold which has an essential disk must be a solid torus, and the essential disk in a solid torus is a fiber.

0.5 Since a semifiber in a bounded 3-manifold $M$ must meet every component of $\partial M$, any essential surface that is disjoint from at least one component of $\partial M$ must be strict.

0.6 Let $M$ be a compact orientable 3-manifold, and let $T$ be a component of $\partial M$ which is a torus. If $F$ is an essential surface in $M$ that meets $T$, then $\partial F \cap T$ is a 1 -manifold in $T$ having no homotopically trivial components. Thus by $0.2, \partial F \cap T$ has a well-defined slope $\sigma \in \mathcal{S}(T)$, which we call the boundary slope of $F$ on $T$.

0.7 We define a knot manifold to be a connected, compact, orientable 3-manifold $M$ such that $\partial M$ is a torus. If $M$ is a knot manifold, we define a (strict) boundary slope of $M$ to be an element of $\mathcal{S}(\partial M)$ which arises as the boundary slope of some bounded (strict) essential surface $F$ in $M$. A theorem of Hatcher's [5; 8] implies that for any given knot manifold $M$, the boundary slopes of $M$ form a finite subset of $\mathcal{S}(\partial M)$. In particular, the strict boundary slopes of $M$ form a finite subset of $\mathcal{S}(\partial M)$.

0.8 If $K$ is a (PL) knot in a closed, orientable 3-manifold $\Sigma$, we shall denote by $V(K)$ a regular neighborhood of $K$, and by $M(K)$ the exterior of $K$, defined by $M=\overline{\Sigma-V(K)}$. Note that $M(K)$ is a knot manifold. Since $V(K)$ and hence $M(K)$ are well-defined up to ambient isotopy in $\Sigma$, and in particular up to homeomorphism, our main results are independent of the choice of $V(K)$. In general we shall implicitly suppose an arbitrary choice of $V(K)$ to have been made, but at one point in Section 3 it will be necessary to be more explicit.

A meridian of $K$ is a nontrivial simple closed curve in the torus $\partial M(K)$ which bounds a disk in $V(K)$. Such a curve exists and is unique up to isotopy. Thus there is a well-defined meridian slope in $\partial M$. A primitive element $\mu$ of $H_{1}(\partial M(K)$; $\mathbb{Z})$ is called a meridian class for $K$ if $\langle\mu\rangle$ is the meridian slope. Thus $K$ has exactly two meridian classes, and they differ by a sign. 
0.9 The knot $K$ will be termed meridionally small if its meridian slope is not a boundary slope for $M(K)$. (In the case where $\Sigma$ is an irreducible nonHaken manifold, meridionally small knots in $\Sigma$ are sometimes called "smallish knots.")

0.10 We define a framing for $K$ to be an ordered basis $(\mu, \lambda)$ for $H_{1}(\partial M(K) ; \mathbb{Z})$ such that $\mu$ is a meridian class.

If $(\mu, \lambda)$ an arbitrary framing for $K$, there is a bijection $v=v_{\mu, \lambda}$ from $\mathcal{S}(\partial M(K))$ to $\mathbb{Q} \cup\{\infty\}$ defined by setting $v(\langle\alpha\rangle)=\omega(\alpha, \lambda) / \omega(\alpha, \mu)$ where $\alpha \in H_{1}(\partial M(K) ; \mathbb{Z})$ is any primitive and $\omega$ denotes homological intersection number. Equivalently, if we write $\alpha=a \mu+b \lambda$, where $a$ and $b$ are relatively prime integers, then $v(\langle\alpha\rangle)=-a / b$.

0.11 Note that if $\left(\mu_{1}, \lambda_{1}\right)$ and $\left(\mu_{2}, \lambda_{2}\right)$ are two framings for the knot $K$, then there exist $h \in \mathbb{Z}$ and $\epsilon \in\{ \pm 1\}$ such that $\mu_{2}=\epsilon \mu_{1}$ and $\lambda_{2}=\lambda_{1}+h \mu_{1}$. It follows that if $\sigma$ is any slope on $\partial M(K)$, and if we set $s_{i}=v_{\mu_{i}, \lambda_{i}}(\sigma)$ for $i=1,2$, then we have

$$
s_{2}=\epsilon s_{1}+h \text {. }
$$

0.12 Now suppose that $M(K)$ is irreducible. If $F$ is a bounded essential surface in $M(K)$, we define the numerical boundary slope of $F$, with respect to a given framing $(\mu, \lambda)$, to be the image of the boundary slope of $F$ under the bijection $v_{\mu, \lambda}: \mathcal{S}(\partial M(K)) \rightarrow \mathbb{Q} \cup\{\infty\}$. In analogy with 0.7 , we define a (strict) numerical boundary slope of $K$ to be a slope on $\partial M(K)$ which arises as the boundary slope of some bounded (strict) essential surface $F$ in $M$. If we denote by $\mathcal{B}_{\mu, \lambda}(K) \subset \mathbb{Q} \cup\{\infty\}$ the set of all numerical boundary slopes of bounded strict essential surfaces in $M(K)$ with respect to the framing $(\mu, \lambda)$, the theorem of Hatcher's quoted in 0.7 implies that $\mathcal{B}_{\mu, \lambda}(K)$ is a finite set.

In particular, if $K$ is meridionally small and has at least one strict boundary slope, then $\mathcal{B}_{\mu, \lambda}(K) \subset \mathbb{Q}$ is a finite, nonempty subset of $Q$; thus in this case $\mathcal{B}_{\mu, \lambda}(K) \subset \mathbb{Q}$ has a greatest element $s_{\max }(K, \mu, \lambda)$ and a least element $s_{\min }(K, \mu, \lambda)$.

The observation 0.11 shows that if $\left(\mu_{1}, \lambda_{1}\right)$ and $\left(\mu_{2}, \lambda_{2}\right)$ are framings of $K$, the sets $\mathcal{B}_{\mu_{1}, \lambda_{1}}(K)$ and $\mathcal{B}_{\mu_{2}, \lambda_{2}}(K)$ differ only by an integer translation and a possible change of sign. In particular, in the case where $K$ is meridionally small and there is at least one strict boundary slope, the diameter $d=s_{\max }(K, \mu, \lambda)-s_{\min }(K, \mu, \lambda)$ of $\mathcal{B}_{\mu, \lambda}(K)$ is independent of the framing $(\mu, \lambda)$ and is therefore an invariant of the knot $K$, which we denote by $d_{K} \in \mathbb{Q}$. If there are no strict boundary slopes for $K$ we set $d_{K}=-\infty$. Thus the invariant $d_{K}$ is defined for every meridionally small knot $K$ in a closed, orientable, irreducible 3-manifold $\Sigma$. 
0.13 A knot $K \subset \Sigma$ is said to be round if some $M(K)$ admits a solid torus as a connected summand. Thus when $M(K)$ is assumed to be irreducible, $K$ is round if and only if $M(K)$ is a solid torus. Note that this implies that $\Sigma$ has a genus-1 Heegaard splitting; that is, $\Sigma$ is homeomorphic either to $S^{2} \times S^{1}$ or to a (possibly trivial) lens space.

0.14 A knot $K^{\prime} \subset \Sigma$ is called a cabling of a knot $K \subset \Sigma$ if there exists a regular neighborhood $U$ of $K$ such that $K^{\prime}$ is a simple closed curve on the boundary of $U$, and the geometric intersection number $q$ of $K^{\prime}$ with the boundary of a meridian disk for the solid torus $U$ is greater than or equal to 2 . We shall refer to the integer $q \geq 2$ as the number of strands of the cable $K^{\prime}$.

Note that according our definition, a knot $L$ which is isotopic in $\Sigma$ to a ( $q$-strand) cabling of $K$ need not itself be a cabling of $K$. However, such a knot $L$ is clearly a ( $q$-strand) cabling of some knot isotopic to $K$.

We define a $q$-strand cable knot in $\Sigma$ to be a knot $K \subset \Sigma$ such that (a) $K$ is not round and (b) $K$ is a $q$-strand cabling of some knot in $\Sigma$. (Note that (b) does not imply (a), since a trivial knot in $S^{3}$ is a $q$-strand cabling of another trivial knot for any $q \geq 2$.) We call $K$ a cable knot if it is a $q$-strand cable knot for some $q \geq 2$.

0.15 A knot $K \subset \Sigma$ is called a generalized iterated torus knot if for some integer $n \geq 0$ there exist knots $K_{0}, K_{1}, \ldots, K_{n}$ in $\Sigma$ such that (i) $K=K_{0}$, (ii) the knot $K_{n}$ is round and (iii) for each $i$ with $0 \leq i \leq n-1$, the knot $K_{i}$ is a cabling of $K_{i+1}$. It follows from the observation made in 0.13 that if $\Sigma$ contains a generalized iterated torus knot whose exterior is irreducible, then $\Sigma$ is either a homeomorph of $S^{2} \times S^{1}$ or a (possibly trivial) lens space.

We are now in a position to give formal statements of our main results.

Theorem A Let $\Sigma$ be a closed, connected, orientable 3-manifold, $K \subset \Sigma$ be a nonround knot, $q \geq 2$ be an integer and $K^{\prime}$ be a $q$-strand cabling of $K$. Suppose that $K^{\prime}$ is meridionally small. Then $K$ is meridionally small, and $d_{K^{\prime}} \geq q^{2} d_{K}$.

This will be proved in Section 3, using foundational material that will be presented in Section 1.

Theorem B Let $\Sigma$ be a closed, connected, orientable 3-manifold such that $\pi_{1}(\Sigma)$ is cyclic. Suppose that $K \subset \Sigma$ is a meridionally small knot. Then either (i) $d_{K} \geq 2$ or (ii) $K$ is a generalized iterated torus knot. 
This will be proved in Section 4 by combining Theorem A with results from [3].

Theorems A and B taken together immediately yield the following:

Corollary C Let $\Sigma$ be a closed, connected, orientable 3-manifold such that $\pi_{1}(\Sigma)$ is cyclic. Suppose that $q \geq 2$ is an integer, and that $K \subset \Sigma$ is a $q$-strand cable knot which is meridionally small. Then either (i) $d_{K} \geq 2 q^{2}$ or (ii) $K$ is a generalized iterated torus knot.

The first author was partially supported by the NSF VIGRE program and by the Chaire de recherche du Canada en algèbre combinatoire et informatique mathématique at the Université du Québec à Montréal. The second author was partially supported by NSF grant DMS 0204142.

\section{Strict essential surfaces in toroidal manifolds}

The main result of this section, Proposition 1.3, will provide a criterion for deciding whether a surface in the cabled knot exterior is strict and essential. This is needed for the proof of Theorem B. The result will be proved in a more general setting: rather than considering only cabled knot exteriors, we shall consider arbitrary compact, irreducible, orientable 3-manifolds that contain essential tori. The result seems likely to be of broader interest in 3-manifold theory.

The following result, which will be used in the proof of Proposition 1.3, is also of more general interest. It says that an essential surface in an irreducible knot manifold is boundary-incompressible in a strong, homotopy-theoretic sense.

1.1 Proposition Suppose that $F$ is a bounded essential surface in an irreducible knot manifold $M$, and suppose that $\alpha$ is a path in $F$ which has its endpoints in $\partial F$ and is fixed-endpoint homotopic in $M$ to a path in $\partial M$. Then $\alpha$ is fixed-endpoint homotopic in $F$ to a path in $\partial F$.

Proof Let $C^{+}$and $C^{-}$denote the upper and lower semicircles in $S^{1}=\partial D^{2}$. The hypothesis implies that there is a map $f: D^{2} \rightarrow M$ such that $\left.f\right|_{C^{+}}$is a reparametrization of $\alpha$ and $f\left(C^{-}\right) \subset \partial M$. We may choose $f$ so that $f^{-1}(\partial M)=C^{-}$and $C^{+}$ is a component of $f^{-1}(F)$, and so that $\left.f\right|_{\left(D^{2}-C^{+}\right)}$is transverse to $F$. Among all maps with these properties, we suppose $F$ to be chosen so as to minimize the number of components of $f^{-1}(F)$. Since $F$ is $\pi_{1}$-injective, the minimality implies that each component of $f^{-1}(F)$ is an arc. Hence some component $A^{+}$of $f^{-1}(F)$ is 
"outermost" in the sense that $A^{+}$is the frontier of a disk $\Delta \subset D^{2}$ with $\Delta \cap \partial D^{2} \subset \operatorname{int} C^{-}$ and $\Delta \cap f^{-1}(F)=A^{+}$. A priori, $A^{+}$may or may not coincide with $C^{+}$.

If $M^{\prime}$ denotes the manifold obtained by splitting $M$ along $F$, and $q: M^{\prime} \rightarrow M$ denotes the quotient map, we may write $\left.f\right|_{\Delta}=q \circ g$ for some map $g: \Delta \rightarrow M^{\prime}$. We set $A^{-}=(\partial \Delta)-\operatorname{int} A^{+}$. We may regard $\left.g\right|_{A^{-}}$as a reparametrization of a path $\beta$ in $q^{-1}(\partial M)$. Since $\partial M$ is a torus, the component $B$ of $q^{-1}(\partial M)$ containing $\beta(I)$ is an annulus. We claim that $\beta$ has both its endpoints in the same component of $\partial B$.

Suppose to the contrary that the endpoints of $\beta$ are in different components of $\partial B$. Then $g$ is homotopic rel $A^{+}$to a map $g^{\prime}: \Delta \rightarrow M^{\prime}$ such that $\left.g^{\prime}\right|_{A^{-}}$is injective and $l^{-}=g^{\prime}\left(A^{-}\right)$is a properly embedded arc in $B$ which meets some core curve of $B$ transversally in a single point. In particular $\left.g^{\prime}\right|_{\partial \Delta}$ is a homotopically nontrivial map of $\partial \Delta$ into $\partial M^{\prime}$, and $g^{\prime}(\partial \Delta) \subset l^{-} \cup F^{\prime}$ for some component $F^{\prime}$ of $q^{-1}(F)$. It now follows from Henderson's version of the loop theorem [7, Theorem III.5] that there is a disk $E \subset M^{\prime}$ such that $\partial E$ is a nontrivial simple closed curve in $\partial M^{\prime}$ and $\partial E \subset l^{-} \cup F^{\prime}$. We cannot have $\partial E \subset F^{\prime}$, since $F$ is $\pi_{1}$-injective in $M$. Hence $\partial E$ must have the form $l^{-} \cup l^{+}$for some properly embedded arc $l^{+}$in $F^{\prime}$.

If $N$ denotes a regular neighborhood of $E$ in $M^{\prime}$ then $R=N \cap F^{\prime}$ is a regular neighborhood of $l^{+}$in $F^{\prime}$, and the closure of (frontier $\left.M^{\prime} N\right)-R$ is a disjoint union of disks $G_{1}$ and $G_{2}$. The surface $F_{1}=(F-q(R)) \cup q\left(G_{1} \cup G_{2}\right)$ is properly embedded in $M$, and is $\pi_{1}$-injective since $F$ is. But since $l$ joins different components of $\partial B$, the component of $\partial F_{1}$ contained in $q(B)$ is a homotopically trivial simple closed curve in $\partial M$. Hence $F_{1}$ is a disk, and by irreducibility it is the frontier of a ball $K \subset M$. We must have either $q(N) \subset K$, in which case $F$ is an annulus contained in the ball $K$, or $q(N) \cap$ int $K=\varnothing$, in which case $F$ is a boundary-parallel annulus. In either case we have a contradiction to the essentiality of $F$. Thus our claim is proved.

We may regard $\left.f\right|_{A^{+}}$as a reparametrization of a path $\alpha_{0}$ in $F$. In the case where $A^{+}=C^{+}$, we may take $\alpha_{0}=\alpha$. Since the endpoints of $\beta$ lie in the same component of $\partial B$, and since $F$ is $\pi_{1}$-injective in $M$, the path $\alpha_{0}$ is fixed-endpoint homotopic in $q(B)$ to a path $\beta_{1}$ in $\partial B \subset \partial F$. This implies the conclusion of the proposition in the case where $A^{+}=C^{+}$. If $A^{+} \neq C^{+}$, we may use the homotopy between $\alpha_{0}$ and $\beta_{1}$ to replace the map $f$ by a map $f_{1}: \Delta \rightarrow M$ which agrees with $f$ on $\Delta$ and maps $D^{2}-\Delta$ into $F$; by perturbing $f_{1}$ slightly we obtain a map $f_{2}$ such that $f_{2}^{-1}(F)$ has fewer components than $f^{-1}(F)$, in contradiction to the minimality property of $f$. Thus the case $A^{+} \neq C^{+}$does not occur, and the proof is complete.

1.2 The proof of the main result of this section, Proposition 1.3, also involves some basic facts about incompressible surfaces in interval bundles over surfaces, which we shall now summarize. 
Suppose that $J$ is an orientable 3 -manifold which is a $[0,1]$-bundle over a surface. We will define a surface in $J$ to be vertical if it is a union of fibers, and to be horizontal if it is everywhere transverse to the fibers. The vertical boundary of $J$ is the inverse image of the boundary of the base of the $I$-bundle $J$ under the projection map.

Any essential vertical annulus in $J$ is the inverse image under the fibration map of an essential simple closed curve in the base.

Suppose that $J$ is a trivial $[0,1]$-bundle and that $F$ is a properly embedded $\pi_{1}$-injective surface in $J$ such that all components of $\partial F$ are contained in the same component $C$ of the $\{0,1\}$-bundle associated to $J$. It follows from Waldhausen [12, Proposition 3.1] that $F$ is isotopic to a horizontal surface by an ambient isotopy that preserves the vertical boundary of $J$, and that each component of $F$ is parallel to a subsurface of $C$.

As a consequence of this fact we observe that if $J$ is a trivial $[0,1]$-bundle, and $F$ is a properly embedded $\pi_{1}$-injective surface in $J$ such that $\partial F$ is contained in the vertical boundary of $J$, then $F$ is isotopic to a horizontal surface by an ambient isotopy that preserves the vertical boundary of $J$.

Suppose that $J$ is a $[0,1]$-bundle and that $A$ is a disjoint union of properly embedded annuli in $J$ none of which is parallel to an annulus contained in the $\{0,1\}$-bundle associated to $J$. It follows from [12, Lemma 3.4] in the case that $J$ is a trivial $[0,1]-$ bundle, and from [1, Lemma 2] in the twisted case that $A$ is isotopic to a vertical surface.

Suppose that $F$ is a properly embedded $\pi_{1}$-injective surface in a $[0,1]$-bundle $J$ such that $\partial F$ is contained in the vertical boundary of $M$. Then $F$ is isotopic to a horizontal surface. This follows from [12, Proposition 3.1 and Proposition 4.1].

1.3 Proposition Let $M$ be a compact orientable irreducible 3-manifold containing an essential torus $T$, let $M^{\prime}$ be the manifold obtained by splitting $M$ along $T$ and let $q: M^{\prime} \rightarrow M$ denote the quotient map. Let $F$ be a connected properly embedded surface in $M$ which is not isotopic to $T$. Then $F$ is a strict essential surface if and only if it is isotopic to a surface $S$ transverse to $T$ such that

(1) each component of $q^{-1}(S)$ is essential in the component of $M^{\prime}$ containing it;

(2) some component of $q^{-1}(S)$ is a strict essential surface in the component of $M^{\prime}$ containing it.

Proof Given a surface $S$ transverse to $T$ we will set $S^{\prime}=q^{-1}(S)$ and $T^{\prime}=q^{-1}(T)$. We let $M_{1}$ denote the manifold obtained by splitting $M$ along $S$ and denote the 
quotient map by $p: M_{1} \rightarrow M$. We let $M_{1}^{\prime}$ denote the manifold obtained by splitting $M^{\prime}$ along $S^{\prime}$, and let $q_{1}: M_{1}^{\prime} \rightarrow M_{1}$ denote the quotient map. We set $S_{1}^{\prime}=q_{1}^{-1}\left(S^{\prime}\right)$.

First suppose that $F$ is a strict essential surface. We will assume that $S$ has been chosen among all surfaces isotopic to $F$ to be transverse to $T$ and to meet $T$ in the minimal number of simple closed curves. We will show that $S$ satisfies conditions (1) and (2).

If $S \cap T=\varnothing$ then $S$ is a strict essential surface in $M^{\prime}$ by 0.5 , so conditions (1) and (2) hold in this case. Thus we may assume that $S \cap T \neq \varnothing$. In particular, since $S$ is connected, no component of $S^{\prime}$ is closed. No component of $S^{\prime}$ can be boundaryparallel since otherwise $S$ would be isotopic to a surface that meets $T$ in fewer simple closed curves. Since $M$ is irreducible it follows in particular that no component of $S^{\prime}$ is a disk. This, together with the $\pi_{1}$-injectivity of $S$, implies that $S^{\prime}$ is $\pi_{1}$-injective in $M^{\prime}$. Hence condition (1) holds for $S$.

To prove that condition (2) holds, assume that every component of $S^{\prime}$ is a semifiber in the component of $M^{\prime}$ containing it. We will show that $M_{1}$ is a [0,1]-bundle, and hence that $S$ is a semifiber, contradicting our supposition that $S$ is strict. By 0.5 our assumption implies that $S^{\prime} \cap T^{\prime} \neq \varnothing$, and hence every component of $M^{\prime}$ contains a component of $S^{\prime}$. The manifold obtained by splitting a component $X$ of $M^{\prime}$ along a component of $S^{\prime}$ is a $[0,1]$-bundle. According to 1.2, all of the other components of $S^{\prime}$ in $X$ are isotopic to horizontal surfaces in this $[0,1]$-bundle. It then follows that the manifold $M_{1}^{\prime}$ has the structure of a $[0,1]$-bundle for which the associated $\{0,1\}$-bundle is the surface $S_{1}^{\prime}$. The vertical boundary of the $[0,1]$-bundle $M_{1}^{\prime}$ is $V=q_{1}^{-1}\left(\partial M^{\prime}\right)$. Observe that $M_{1}$ is a quotient of $M_{1}^{\prime}$ obtained by identifying pairs of components of $V$. To obtain the required [0,1]-bundle structure on $M_{1}$ it suffices to observe that the gluing maps are isotopic to fiber-preserving maps with respect to the $[0,1]$-bundle structures on the components of $V$. This is because any homeomorphism between two trivial $[0,1]$-bundles over $S^{1}$ is isotopic to a fiber-preserving map.

As a preliminary to proving the converse we observe that, since $T$ is $\pi_{1}$-injective and $M$ is irreducible, any properly embedded disk in $M^{\prime}$ having its boundary contained in $T^{\prime}$ must be boundary-parallel in $M^{\prime}$.

For the proof of the converse we assume that conditions (1) and (2) hold for the surface $S$. No component of $S^{\prime}$ can be a $2-$ sphere. Hence if $S$ had a $2-$ sphere component then some component of $S^{\prime}$ would be a disk whose boundary is contained in $T^{\prime}$. Since any such disk is boundary-parallel, this would contradict condition (1). Thus $S$ has no 2-sphere components.

Suppose that $S$ fails to be $\pi_{1}$-injective. Then we may choose a compressing disk $D$ for $S$ which is transverse to $T$ and meets $T$ in the minimal number of components. 
Since $T$ is $\pi_{1}$-injective, the minimality implies that all components of $D \cap T$ must be arcs. Since $S^{\prime}$ is $\pi_{1}$-injective in $M^{\prime}$ according to (1), we have $D \cap T \neq \varnothing$. An outermost $\operatorname{arc} \beta$ of $D \cap T$ is the image under $q$ of an $\operatorname{arc}$ in $\partial M^{\prime}$ which is fixed-endpoint homotopic in $M^{\prime}$ to an arc $\alpha$ in $S^{\prime}$. It follows from an application of Proposition 1.1, with $M$ replaced by $M^{\prime}$ and $F$ by $S^{\prime}$, that $\alpha$ is the frontier of a disk $E_{1}$ in $S^{\prime}$. The subdisk of $D$ cobounded by $\alpha$ and $\beta$ is the image of a disk $E_{2}$ in $M^{\prime}$. The union of $E_{1}$ and $E_{2}$ is a properly embedded disk in $M^{\prime}$ having its boundary contained in $T^{\prime}$, and must therefore be a boundary-parallel disk in $M^{\prime}$. It now follows that $D$ is isotopic to a disk that has fewer components of intersection with $T$, contradicting our choice of $D$. This shows that $S$ is $\pi_{1}$-injective.

Suppose that $S$ is boundary-parallel. We will show that some component of $S^{\prime}$ is boundary-parallel, contradicting condition (1). There is a submanifold $P$ of $M$ whose frontier is $S$ such that the pair $(P, S)$ is homeomorphic to $(S \times I, S \times\{1\})$. If $T \cap P=\varnothing$ then it is immediate that $S^{\prime}$ is boundary-parallel in $M^{\prime}$. Since $S$ is $\pi_{1}$-injective, if some component of $T \cap S$ is homotopically trivial in $M$ then it must bound a disk in $S$. A minimal disk in $S$ bounded by a component of $T \cap S$ is the image under $q$ of a properly embedded disk $D \subset M^{\prime}$ with $\partial D \subset T^{\prime}$. Since $D$ must be boundary-parallel in $M^{\prime}$, it is the required boundary-parallel component of $S^{\prime}$. If every component of $T \cap S=\partial(T \cap P)$ is homotopically nontrivial in $M$ then since $T$ is $\pi_{1}$-injective, $T \cap P$ is $\pi_{1}$-injective. It therefore follows from 1.2 that every component of $T \cap P$ is parallel to a subsurface of $S$. This implies that some component of $S^{\prime}$ is boundary-parallel in $M^{\prime}$. This completes the proof that $S$ is essential.

To show that $S$ is strict assume, to the contrary, that the manifold $M_{1}$ is a $[0,1]-$ bundle and that the associated $\{0,1\}$-bundle is $S_{1}=p^{-1}(S)$. Then $T_{1}=p^{-1}(T)$ is a disjoint union of annuli in $M_{1}$ whose boundary components are contained in the $\{0,1\}$-bundle $S_{1}$. If any of these annuli were parallel into $S_{1}$ it would imply that some component of $S^{\prime}$ is a boundary-parallel annulus in $M^{\prime}$, contradicting condition (1). It therefore follows from 1.2 that each component of $T_{1}$ is isotopic to a vertical annulus in the $[0,1]$-bundle $M_{1}$. Therefore the manifold $M_{1}^{\prime}$, which we can think of as being obtained by splitting $M_{1}$ along $T_{1}$, is a [0,1]-bundle whose associated $\{0,1\}$-bundle is $S_{1}^{\prime}$. This contradicts condition (2). Thus $S$ is a strict essential surface in $M$.

\section{Cable spaces}

We have said that Proposition 1.3 can be used to identify strict essential knots in cabled knot exteriors. This is because the exterior of a cabling $K^{\prime}$ of a knot $K$ can be regarded as being constructed from $M(K)$ by gluing a "cable space" to its boundary. We begin 
this section with a definition and some observations related to this construction, and then prove Lemma 2.3, which provides a wealth of essential surfaces in a cable space.

2.1 We define a cable space to be a Seifert fibered manifold over an annulus with one singular fiber. Note that a cable space has exactly three isotopy classes of essential vertical annuli; one has a boundary curve on each boundary torus of the cable space and the other two have both boundary curves on the same boundary torus.

2.2 Let $K$ be a knot in a closed, orientable, connected 3-manifold and $K^{\prime}$ be a $q$-strand cabling of $K$ for some $q \geq 2$. It follows from the definition of a cable in 0.14 that $K$ has a regular neighborhood $V$ such that $K^{\prime}$ is contained in a torus $T \subset$ int $V$ which is boundary-parallel in $V$, and $K^{\prime}$ has geometric intersection number $q$ with the boundary of a meridian disk for the solid torus $V$. We shall call a neighborhood $V$ with these properties an enveloping solid torus for the cabling $K^{\prime}$ of $K$.

If $V$ is an enveloping solid torus for a $q$-strand cabling $K^{\prime}$ of a knot $K$ then $V$ admits a Seifert fibration over a disk in which $K^{\prime}$ is a regular fiber, $K$ is the only singular fiber, and the order of this singular fiber is $q$. Hence if $W \subset$ int $V$ is a regular neighborhood of $K^{\prime}$ disjoint from $K$, then $N=\overline{V-W}$ admits a Seifert fibration over an annulus which has exactly one singular fiber, and the order of the singular fiber is $q$. In particular, $N$ is a cable space.

2.3 Lemma Suppose that $N$ is a cable space (see 2.1) with boundary tori $T_{1}$ and $T_{2}$. Let $\iota^{j}$ denote the inclusion map from $T_{j}$ to $N$. Then there exists a bijection $\phi=\phi_{N, T_{1}, T_{2}}: \mathcal{S}\left(T_{1}\right) \rightarrow \mathcal{S}\left(T_{2}\right)$ having the following properties:

(i) if $\alpha_{j}$ is a primitive element of $H_{1}\left(T_{j} ; \mathbb{Z}\right) \subset H_{1}\left(T_{j} ; \mathbb{Q}\right)$ for $j=1,2$, and if $\phi\left(\left\langle\alpha_{1}\right\rangle\right)=\left\langle\alpha_{2}\right\rangle$, then $\iota_{\sharp}^{2}\left(\alpha_{2}\right)$ is a rational multiple of $\iota_{\sharp}^{1}\left(\alpha_{1}\right)$ in $H_{1}(N ; \mathbb{Q})$;

(ii) for each slope $\sigma$ on $T_{1}$ there exists a connected essential surface in $N$ which has nonempty intersection with both $T_{1}$ and $T_{2}$ and has $\sigma$ and $\phi(\sigma)$ as boundary slopes.

Proof We identify $H_{1}(\partial N ; \mathbb{Q})$ with $H_{1}\left(T_{1} ; \mathbb{Q}\right) \oplus H_{1}\left(T_{2} ; \mathbb{Q}\right)$. The cable space $N$ may be decomposed as the union of homeomorphic copies of $D^{2} \times S^{1}$ and $S^{1} \times$ $S^{1} \times I$, meeting along an annulus. A Mayer-Vietoris computation shows that $\iota_{\sharp}^{j}$ is an isomorphism $H_{1}\left(T_{j} ; \mathbb{Q}\right) \rightarrow H_{1}(N ; \mathbb{Q})$ for $j=1,2$. If $\alpha_{1}$ is a primitive element of $H_{1}\left(T_{1} ; \mathbb{Z}\right) \subset H_{1}\left(T_{1} ; \mathbb{Q}\right)$ then up to sign there is a unique primitive element $\alpha_{2}$ of $H_{1}\left(T_{2} ; \mathbb{Z}\right) \subset H_{1}\left(T_{2} ; \mathbb{Q}\right)$ which is a rational multiple of $\left(\iota_{\sharp}^{2}\right)^{-1} \circ \iota_{\sharp}^{1}\left(\alpha_{1}\right)$. We then define the map $\phi$ by setting $\phi\left(\left\langle\alpha_{1}\right\rangle\right)=\left\langle\alpha_{2}\right\rangle$; this is a well-defined bijection since every slope 
on $T_{j}$ may be written in the form $\langle\alpha\rangle$, where $\alpha$ is determined up to sign by the slope. Property (i) of $\phi$ is immediate from the definition.

To prove Property (ii), let $\sigma$ be any slope on $T_{1}$. Write $\sigma=\left\langle\alpha_{1}\right\rangle$ and $\phi(\sigma)=\left\langle\alpha_{2}\right\rangle$ where $\alpha_{1} \in H_{1}\left(T_{1} ; \mathbb{Z}\right)$ and $\alpha_{2} \in H_{1}\left(T_{2} ; \mathbb{Z}\right)$ are primitive elements such that the sum $m \iota_{\sharp}^{1}\left(\alpha_{1}\right)+n \iota_{\sharp}^{2}\left(\alpha_{2}\right)=0$ for some relatively prime integers $m$ and $n$. By the long exact homology sequence of $(N, \partial N)$ there is a class $c$ in $H_{2}(N, \partial N ; \mathbb{Z})$ whose image under the boundary map $\partial: H_{2}(N, \partial N ; \mathbb{Z}) \rightarrow H_{1}(\partial N ; \mathbb{Z})$ is $m \alpha_{1} \oplus n \alpha_{2}$. It follows from the proof of [6, Lemma 6.6] that there is an oriented essential surface $S$ which represents the homology class $c$. Since $\partial(c) m \alpha_{1} \oplus n \alpha_{2}$, and since $S$ is essential and $T_{1}$ and $T_{2}$ are tori, every component of $\partial S \cap T_{1}$ has slope $\sigma$ and every component of $\partial S \cap T_{2}$ has slope $\phi(\sigma)$.

Since $\partial(c) \neq 0$, some component $S_{0}$ of $S$ must represent a class $c_{0} \in H_{2}(N, \partial N ; \mathbb{Z})$ such that $\partial\left(c_{0}\right) \neq 0$. Note that $S_{0} \cap T_{2}$ is nonempty since otherwise $\partial\left(c_{0}\right)$ would be a nonzero multiple of $\alpha_{1}$, which is impossible since the image of $\alpha_{1}$ in $H_{1}(N ; \mathbb{Z})$ has infinite order. Similarly $S_{0}$ must have nonempty intersection with $T_{1}$. Furthermore, since $S_{0}$ is a component of $S$ it is essential and has boundary slopes $\sigma$ and $\phi(\sigma)$.

\section{The effect of cabling on strict boundary slopes}

The goal of this section is to prove Theorem A of the Introduction.

3.1 In the next two lemmas, 3.2 and 3.3, we shall consider a knot $K$ in a closed, connected, orientable 3-manifold $\Sigma$ and a cabling $K^{\prime}$ of $K$ with $q \geq 2$ strands. We shall denote by $V$ an enveloping solid torus for the cabling $K^{\prime}$ and by $W$ a regular neighborhood of $K^{\prime}$ that is contained in $V$ and disjoint from $K$. We shall make the explicit choices $V(K)=V$ and $V\left(K^{\prime}\right)=W$ for the regular neighborhoods of $K$ and $K^{\prime}$ (see 0.8 ). We shall set $N=\overline{V-W}$, so that $N$ is a cable space by 2.2. We set $T_{1}=\partial V=\partial M(K)$ and $T_{2}=\partial W=\partial M\left(K^{\prime}\right)$. Thus $T_{1}$ and $T_{2}$ are the boundary tori of the cable space $N$. We let $\phi=\phi_{N, T_{1}, T_{2}}: \mathcal{S}\left(T_{1}\right) \rightarrow \mathcal{S}\left(T_{2}\right)$ denote the bijection given by Lemma 2.3 .

3.2 Lemma Suppose that $K$ is a knot in a closed, connected, orientable 3-manifold $\Sigma$, and that $K^{\prime}$ is a cabling of $K$ with $q \geq 2$ strands. Let $V, W, N, T_{1}, T_{2}$ and $\phi$ be defined as in 3.1. Let $v=v_{\mu, \lambda}: \mathcal{S}\left(T_{1}\right) \rightarrow \mathbb{Q} \cup\{\infty\}$ and $v^{\prime}=v_{\mu^{\prime}, \lambda^{\prime}}: \mathcal{S}\left(T_{2}\right) \rightarrow \mathbb{Q} \cup\{\infty\}$ be the bijections given by 0.10 , Then there exist constants $u \in \mathbb{Q}$ and $\epsilon \in\{ \pm 1\}$ such that for every $\sigma \in \mathcal{S}\left(T_{1}\right)$ we have

$$
v^{\prime}(\phi(\sigma))=\epsilon q^{2} v(\sigma)+u .
$$

Here the right-hand side is interpreted to be $\infty$ if $v(\sigma)=\infty$. 
Proof Since $V$ is an enveloping solid torus for the $q$-strand cabling $K^{\prime}$ of $K$, there is a meridian disk $D$ for the solid torus $V$ which meets $K$ transversally in one point, and meets $K^{\prime}$ transversally in $q$ points. Furthermore, the intersections of $D$ with $K^{\prime}$ are all consistently oriented, in the sense that if $\omega$ is a transverse orientation to $D$ in $V$, the orientations of $K^{\prime}$ induced by $\omega$ at the different points of $D \cap K^{\prime}$ all coincide. By choosing $D$ to be in standard position with respect to $W$ we may arrange that $D \cap W$ consists of $q$ meridian disks in $W$, each of which contains a unique point of $D \cap K^{\prime}$. Now $P=D \cap N$ is a planar surface whose boundary consists of one meridian curve in $T_{1}=\partial V$ and $q$ meridian curves in $T_{2}=\partial W$.

We identify $H_{1}(\partial N ; \mathbb{Z})$ with $H_{1}\left(T_{1} ; \mathbb{Z}\right) \oplus H_{1}\left(T_{2} ; \mathbb{Z}\right)$. Since the intersections of $D$ with $K^{\prime}$ are all consistently oriented, we may orient $P$ in such a way that the class $[P] \in H_{2}(N, \partial N ; \mathbb{Z})$ satisfies

$$
\partial[P]=\mu+\zeta q \mu^{\prime}
$$

for some $\zeta \in\{ \pm 1\}$.

For $i=1,2$ and for every $\alpha \in H_{1}\left(T_{i} ; \mathbb{Z}\right)$, let us denote by $\bar{\alpha}$ the image of $\alpha$ under the natural homomorphism $H_{1}(\partial N ; \mathbb{Z}) \rightarrow H_{1}(N ; \mathbb{Q})$. It follows from (3.2.1) that

$$
\bar{\mu}=-\zeta q \bar{\mu}^{\prime} .
$$

We let $\theta \in\{ \pm 1\}$ denote the homological intersection number of $\lambda^{\prime}$ with $\mu^{\prime}$ in $T_{2}$. Then by (3.2.1), the homological intersection number of $\bar{\lambda}^{\prime}$ with $[P]$ in $N$ equals $\zeta \theta q$.

We saw in the proof of Lemma 2.3 that the inclusion homomorphism from $H_{1}\left(T_{i} ; \mathbb{Q}\right)$ into $H_{1}(N ; \mathbb{Q})$ is an isomorphism for $i=1,2$. In particular, $\bar{\lambda}$ and $\bar{\mu}$ form a basis for $H_{1}(N ; \mathbb{Q})$. Let us write $\bar{\lambda}^{\prime}=t \bar{\mu}+h \bar{\lambda}$ for some $t, h \in \mathbb{Q}$. If $\eta \in\{ \pm 1\}$ denotes the homological intersection number of $\lambda$ with $\mu$ in $T_{1}$, it follows again from (3.2.1) that the homological intersection number of $\bar{\lambda}^{\prime}$ with $[P]$ in $N$ is $\eta h$. Hence $\eta h=\zeta \theta q$, and therefore

$$
\bar{\lambda}^{\prime}=t \bar{\mu}+\zeta \theta \eta q \bar{\lambda}
$$

We shall show that the lemma holds if we set $\epsilon=\eta \theta \in\{ \pm 1\}$ and $u=\zeta q t \in \mathbb{Q}$.

Consider any element $\sigma$ of $\mathcal{S}\left(T_{1}\right)$. We may write $\sigma=\langle\alpha\rangle$ for some primitive element $\alpha=a \mu+b \lambda$ of $H_{1}\left(T_{1} ; \mathbb{Z}\right)$, where $a$ and $b$ are relatively prime integers. We have $v(\sigma)=-a / b$. Likewise, if we write $\phi(\sigma)=\left\langle\alpha^{\prime}\right\rangle$ for some primitive $\alpha^{\prime}=a^{\prime} \mu^{\prime}+b^{\prime} \lambda^{\prime}$ in $H_{1}\left(T_{1} ; \mathbb{Z}\right)$, then $v^{\prime}(\phi(\sigma))=-a^{\prime} / b^{\prime}$. Using (3.2.2) and (3.2.3), we find that

$$
\bar{\alpha}^{\prime}=a^{\prime} \bar{\mu}^{\prime}+b^{\prime} \bar{\lambda}^{\prime}=-\frac{\zeta a^{\prime} \bar{\mu}}{q}+b^{\prime}(t \bar{\mu}+\zeta \theta \eta q \bar{\lambda})=\left(\frac{b^{\prime} q t-\zeta a^{\prime}}{q}\right) \bar{\mu}+\zeta \theta \eta b^{\prime} q \bar{\lambda} .
$$


According to 2.3 (i), $\bar{\alpha}$ is a rational multiple of $\bar{\alpha}^{\prime}$, say $\bar{\alpha}=r \bar{\alpha}^{\prime}$. Hence

$$
\bar{\alpha}=\left(\frac{b^{\prime} q t-\zeta a^{\prime}}{q}\right) r \bar{\mu}+\zeta \theta \eta b^{\prime} q r \bar{\lambda}
$$

On the other hand, we have

$$
\bar{\alpha}=a \bar{\mu}+b \bar{\lambda},
$$

and since $\bar{\lambda}$ and $\bar{\mu}$ form a basis for $H_{1}(N ; \mathbb{Q})$, it follows that

$$
a=\left(\frac{b^{\prime} q t-\zeta a^{\prime}}{q}\right) r \quad \text { and } \quad b=\zeta \theta \eta b^{\prime} q r .
$$

Hence $v(\sigma)=-\frac{a}{b}=-\frac{\left(b^{\prime} q t-\zeta a^{\prime}\right) / q}{\zeta \theta \eta b^{\prime} q}=\frac{\theta \eta}{q^{2}} \frac{a^{\prime}}{b^{\prime}}-\frac{\zeta \eta \theta t}{q}=\frac{\theta \eta}{q^{2}} \nu^{\prime}(\phi(\sigma))-\frac{\zeta \eta \theta t}{q}$.

This gives the required equality

$$
v^{\prime}(\phi(\sigma))=\theta \eta q^{2} v(\sigma)+\zeta q t=\epsilon q^{2} v(\sigma)+u
$$

3.3 Lemma Suppose that $K$ is a knot in a closed, connected, orientable 3-manifold $\Sigma$, and that $K^{\prime}$ is a cabling of $K$ with $q \geq 2$ strands. Let $V, W, N, T_{1}, T_{2}$ and $\phi$ be defined as in 3.1. Suppose that $\sigma \in \mathcal{S}\left(T_{1}\right)=\mathcal{S}(\partial M(K))$ is a strict boundary slope for $K$. Then $\phi(\sigma) \in \mathcal{S}\left(T_{2}\right)=\mathcal{S}\left(\partial M\left(K^{\prime}\right)\right)$ is a strict boundary slope for $K^{\prime}$.

Proof Since $\sigma$ is a strict boundary slope for $K$, there is a connected strict essential surface $F \subset M(K)$ having boundary slope $\sigma$. On the other hand, by Lemma 2.3, there is a connected essential surface $E \subset N$, having nonempty intersection with both $T_{1}$ and $T_{2}$ and having $\sigma$ and $\phi(\sigma)$ as boundary slopes. Let $m$ and $n$ denote, respectively, the numbers of components of $\partial F$ and $\partial E$. Let $E^{*} \subset N$ be an essential surface with $m$ components, each isotopic to $E$ in $N$, and let $F^{*} \subset M$ be a strict essential surface with $n$ components, each isotopic to $F$ in $M$. Then $\partial E^{*}$ and $\partial F^{*}$ are both 1 -manifolds in $T_{1}$ with slope $\sigma$, and each of them has $m n$ components. Hence after varying $E$ within its isotopy class we may assume that $\partial E=\partial F$. This means that $F^{\prime}=E \cup F$ is a properly embedded surface in $M\left(K^{\prime}\right)=M(K) \cup N$ transverse to $T_{1}$. Since $E=F^{\prime} \cap N$ is an essential surface in $N$, and $F=F^{\prime} \cap M(K)$ is a strict essential surface in $M\left(K^{\prime}\right)$, it follows from Proposition 1.3 that $F^{\prime}$ is a strict essential surface in $M\left(K^{\prime}\right)$. As the boundary slope of $F^{\prime}$ is clearly equal to $\phi(\sigma) \in \mathcal{S}\left(T_{2}\right)=\mathcal{S}\left(\partial M\left(K^{\prime}\right)\right)$, it follows that $\phi(\sigma)$ is a boundary slope for $K^{\prime}$.

Proof of Theorem A We are given a nonround knot $K$ in a closed, connected, orientable 3-manifold $\Sigma$ and a cabling $K^{\prime}$ of $K$ with $q \geq 2$ strands such that $K^{\prime}$ is meridionally small. We define $V, W, N, T_{1}, T_{2}$ and $\phi$ as in 3.1. We also choose 
framings $(\mu, \lambda)$ and $\left(\mu^{\prime}, \lambda^{\prime}\right)$ for $K$ and $K^{\prime}$, and let $v=v_{\mu, \lambda}: \mathcal{S}\left(T_{1}\right) \rightarrow \mathbb{Q} \cup\{\infty\}$ and $v^{\prime}=v_{\mu^{\prime}, \lambda^{\prime}}: \mathcal{S}\left(T_{2}\right) \rightarrow \mathbb{Q} \cup\{\infty\}$ be the bijections given by 0.10 . We denote by $\epsilon \in\{ \pm 1\}$ and $u \in \mathbb{Q}$ the constants given by Lemma 3.2.

We must first show that $K$ is meridionally small, ie, that $M(K)$ is irreducible and that the meridian slope of $K$ is not a strict boundary slope. If $S \subset$ int $M(K)$ is a 2-sphere, then $S$ bounds a ball $B$ in the interior of $M\left(K^{\prime}\right)=M(K) \cup N$. Since $N$ is connected and disjoint from $S$, we must have either $N \subset$ int $B$ or $N \cap B=\varnothing$. But $N \subset$ int $B$ would imply $\partial M\left(K^{\prime}\right) \subset B$, a contradiction. Hence $N \cap B=\varnothing$ and therefore $B \subset M(K)$. This shows that $M(K)$ is irreducible.

Next note that, according to Lemma 3.2, we have $v^{\prime}(\phi(\langle\mu\rangle))=\epsilon q^{2} v(\langle\mu\rangle)+u$, where $v(\langle\mu\rangle)=\infty$. Hence $v^{\prime}(\phi(\langle\mu\rangle))=\infty$, that is, $\phi(\langle\mu\rangle)=\mu^{\prime}$. If $\langle\mu\rangle$ were a strict boundary slope for $K$, Lemma 3.3 would now imply that the meridian slope $\left\langle\mu^{\prime}\right\rangle$ is a strict boundary slope for $K^{\prime}$, a contradiction to the hypothesis that $K^{\prime}$ is meridionally small. Hence the meridian slope $\langle\mu\rangle$ is not a strict boundary slope for $K$. This completes the proof that $K$ is meridionally small.

It remains to show that $d_{K^{\prime}} \geq q^{2} d_{K}$. By definition of $d_{K}$, there exist strict boundary slopes $\sigma$ and $\tau$ for $K$ such that

$$
v(\sigma)-v(\tau)=d_{K}
$$

According to Lemma 3.3, the slopes $\sigma^{\prime}=\phi(\sigma)$ and $\tau^{\prime}=\phi(\tau)$ are strict boundary slopes for $K^{\prime}$. But from Lemma 3.2 we have

$$
\left|v^{\prime}\left(\sigma^{\prime}\right)-v^{\prime}\left(\tau^{\prime}\right)\right|\left|\left(\epsilon q^{2} v(\sigma)+u\right)-\left(\epsilon q^{2} v(\tau)+u\right)\right|=q^{2} d_{K} .
$$

This shows that $d_{K^{\prime}} \geq q^{2} d_{K}$.

\section{A lower bound for $d_{K}$}

The goal of this section is to prove Theorem B of the Introduction.

4.1 Proposition Suppose that $K$ is a knot in a closed, connected, orientable 3manifold $\Sigma$. Then for some integer $n \geq 0$ there exist knots $K_{0}, K_{1}, \ldots, K_{n}$ in $\Sigma$ such that (i) $K=K_{0}$, (ii) $K_{n}$ is not a cable knot and (iii) for each $i$ with $0 \leq i \leq n-1$, the knot $K_{i}$ is a cabling of $K_{i+1}$.

4.2 Remark According to our definition of a cable knot in 0.14, it may happen that the knot $K_{n}$ given by Proposition 4.1 is round. If $K_{n}$ is round then it follows from the definition that $K$ is a generalized iterated torus knot. 
Proof of Proposition 4.1 Suppose that $K$ is a knot in a closed, connected, orientable 3-manifold $\Sigma$. Set $M=M(K)$. Since $M$ is a compact, irreducible, orientable 3-manifold, it follows from Haken's finiteness theorem [6, Lemma 1.32] that there is an integer $\Theta>0$ with the following property: if $F_{1}, \ldots, F_{\Theta}$ are disjoint, closed, connected, orientable surfaces of strictly positive genus in int $M$ such that the homomorphism $\pi_{1}\left(F_{i}\right) \rightarrow \pi_{1}(M)$ is injective for $i=1, \ldots, \Theta$, then the closure of some component of $M-\left(F_{1} \cup \cdots \cup F_{\Theta}\right)$ is homeomorphic to $F \times[0,1]$ for some closed surface $F$.

Now assume that the conclusion of Proposition 4.1 does not hold. We shall recursively construct knots $K_{0}, \ldots, K_{\Theta}$ in $\Sigma$ and regular neighborhoods $V_{i}$ of the $K_{i}$ in such a way that for $i=0, \ldots, \Theta-1$ these conditions hold: (i) $K_{i}$ is a cabling of $K_{i+1}$, (ii) $V_{i+1}$ is an enveloping solid torus (in the sense of 2.2) for the cabling $K_{i}$ of $K_{i+1}$ and (iii) $V_{i}$ is contained in the interior of $V_{i+1}$ and is disjoint from $K_{i+1}$.

We set $K_{0}=K$ and set $V_{0}=V(K)$. Now suppose that for a given $m \in\{0, \ldots, \Theta-1\}$ we have defined $K_{0}, \ldots, K_{m}$ and $V_{0}, \ldots, V_{m}$ so that conditions (i)-(iii) hold for every $i$ with $0 \leq i<m$. (This is of course vacuously true when $m=0$.) We need to define $K_{m+1}$ and $V_{m+1}$ so that (i)-(iii) hold for $i=m$.

If $K_{m}$ were not a cable knot, then since condition (i) holds for $0 \leq i<m$, the conclusion of Proposition 4.1 would hold with $n=m$. As we have assumed that this conclusion does not hold, $K_{m}$ is a cable knot. In particular, $K_{m}$ is a cabling of some knot $K^{*}$ in $\Sigma$. Let $V^{*}$ be an enveloping solid torus for the cabling $K_{m}$ of $K^{*}$, and let $W^{*}$ be a regular neighborhood of $K_{m}$ which is contained in the interior of $V^{*}$ and is disjoint from $K_{m+1}$. Since $W^{*}$ and $V_{m}$ are both regular neighborhoods of $K_{m}$ in $\Sigma$, there is a homeomorphism $h: \Sigma \rightarrow \Sigma$, isotopic to the identity by an isotopy fixing $K_{m}$, such that $h\left(W^{*}\right)=V_{m}$. The knot $K_{m+1}=h\left(K^{*}\right)$ and the solid torus $V_{m+1}=h\left(V^{*}\right)$ then have the required properties. This completes the recursive construction.

If $K_{\Theta}$ were not a cable knot, then since condition (i) holds for $0 \leq i<\Theta$, the conclusion of Proposition 4.1 would hold with $n=\Theta$. As we have assumed that this conclusion does not hold, $K_{\Theta}$ is a cable knot. In particular, $K_{\Theta}$ is not round.

For $i=0, \ldots, \Theta-1$ set $N_{i}=\overline{V_{i+1}-V_{i}}$. According to 2.2, each $N_{i}$ is a cable space.

For $i=0, \ldots, \Theta$, set $T_{i}=\partial V_{i}$. Note that the $T_{i}$ are disjoint tori contained in int $M(K)$. Set $\mathcal{T}=T_{1} \cup \cdots \cup T_{\Theta}$. Note that the closures of the components of $M(K)-\mathcal{T}$ are $N_{0}, \ldots, N_{\Theta-1}$ and $\overline{\Sigma-V_{\Theta}}$.

We distinguish two cases. First suppose that for some $i \in\{0, \ldots, \Theta\}$ the inclusion homomorphism $\pi_{1}\left(T_{i}\right) \rightarrow \pi_{1}\left(\overline{\Sigma-V_{0}}\right)$ has a nontrivial kernel. Then by [6, Lemma 6.1], there is a disk $D \subset M(K)$ such that $D \cap \mathcal{T}=\partial D$, and such that $\partial D$ does not bound a 
disk in $\mathcal{T}$. Let $Z$ denote the component of $M(K)-\mathcal{T}$ that contains int $D$, so that $D$ is an essential properly embedded disk in $\bar{Z}$. If $\bar{Z}=\overline{\Sigma-V_{\Theta}}$, then $M\left(K_{\Theta}\right) \cong \overline{\Sigma-V_{\Theta}}$ contains an essential disk and therefore has a solid torus as a connected summand; this contradicts the fact that $K_{\Theta}$ is not round. If $\bar{Z}=N_{i}$ for some $i \in\{0, \Theta-1\}$ we again obtain a contradiction, because the cable space $N_{i}$ is a Seifert fibered space with two boundary components, and the only Seifert fibered space that contains an essential disk is the solid torus.

There remains the case that $\pi_{1}\left(T_{i}\right) \rightarrow \pi_{1}\left(\overline{\Sigma-V_{0}}\right)$ is injective for each $i \in\{0, \ldots, \Theta\}$. The defining property of $\Theta$ then implies that the closure of some component $Z$ of $M(K)-\mathcal{T}$ is homeomorphic to $T^{2} \times[0,1]$. We cannot have $\bar{Z}=\overline{\Sigma-V_{\Theta}}$, since $\overline{\Sigma-V_{\Theta}}$ has connected boundary. Hence we must have $\bar{Z}=N_{i}$ for some $i \in\{0, \Theta-1\}$. But since the cable space $N_{i}$ is a Seifert fibered space over an annulus with a singular fiber, the fundamental group of either component of $\partial N_{i}$ is mapped by the inclusion homomorphism onto a proper subgroup of $\pi_{1}\left(N_{i}\right)$; hence $N_{i}$ cannot be homeomorphic to $T^{2} \times[0,1]$, and we have a contradiction in this case as well.

Proof of Theorem B Given a closed, connected, orientable 3-manifold $\Sigma$ such that $\pi_{1}(\Sigma)$ is cyclic, and a meridionally small knot $K \subset \Sigma$, we must show that either (i) $d_{K} \geq 2$ or (ii) $K$ is a generalized iterated torus knot.

Let $n \geq 0$ be the integer and $K_{0}, K_{1}, \ldots, K_{n}$ the knots given by Proposition 4.1. For each $i, 0 \leq i \leq n-1$, the knot $K_{i}$ is a $q_{i+1}$-strand cabling of $K_{i+1}$ for some $q_{i} \geq 2$.

If $K_{m}$ is round for some $m \leq n$, then it follows from Remark 4.2 that $K$ is a generalized iterated torus knot. Thus conclusion (ii) holds in this case.

Now suppose that none of the knots $K_{0}, \ldots, K_{n}$ is round. By $n$ successive applications of Theorem A we see that each of the $K_{i}$ is meridionally small, and that for $i=$ $0, \ldots, n-1$. Hence

$$
d_{K}=d_{K_{0}} \geq q_{1}^{2} \cdots q_{n}^{2} d_{K_{n}} .
$$

On the other hand, since $\pi_{1}(\Sigma)$ is cyclic, and since $K_{n}$ is meridionally small and is not a round knot or a cable knot, it follows from Theorem 1.1 of [3] that $d_{K_{n}} \geq 2$. Hence

$$
d_{K} \geq 2 q_{1}^{2} \cdots q_{n}^{2} \geq 2,
$$

and so (i) holds. 


\section{References}

[1] M Brittenham, Exceptional Seifert-fibered spaces and Dehn surgery on 2-bridge knots, Topology 37 (1998) 665-672 MR1604911

[2] M Culler, P B Shalen, Bounded, separating, incompressible surfaces in knot manifolds, Invent. Math. 75 (1984) 537-545 MR735339

[3] M Culler, P B Shalen, Boundary slopes of knots, Comment. Math. Helv. 74 (1999) 530-547 MR1730656

[4] M Culler, P B Shalen, Knots with only two strict essential surfaces, from: "Proceedings of the Casson Fest", (C Gordon, Y Rieck, editors), Geom. Topol. Monogr. 7 (2004) 335-430 MR2172490

[5] A E Hatcher, On the boundary curves of incompressible surfaces, Pacific J. Math. 99 (1982) 373-377 MR658066

[6] J Hempel, 3-Manifolds, Princeton University Press, Princeton, N. J. (1976) MR0415619

[7] D W Henderson, Extensions of Dehn's lemma and the loop theorem, Trans. Amer. Math. Soc. 120 (1965) 448-469 MR0187233

[8] W Jaco, E Sedgwick, Decision problems in the space of Dehn fillings, Topology 42 (2003) 845-906 MR1958532

[9] B Klaff, Boundary slopes of knots in closed manifolds with cyclic fundamental group, $\mathrm{PhD}$ thesis, University of Illinois at Chicago (2003)

[10] L P Neuwirth, Knot groups, Annals of Mathematics Studies, No. 56, Princeton University Press, Princeton, N.J. (1965) MR0176462

[11] P B Shalen, Representations of 3-manifold groups, from: "Handbook of geometric topology”, North-Holland, Amsterdam (2002) 955-1044 MR1886685

[12] F Waldhausen, On irreducible 3-manifolds which are sufficiently large, Ann. of Math. (2) 87 (1968) 56-88 MR0224099

Department of Mathematics, University of Texas at Austin

1 University Station, Austin, TX 78741, USA

Department of Mathematics, Statistics, and Computer Science (M/C 249)

University of Illinois at Chicago, 851 S. Morgan St., Chicago, IL 60607-7045, USA

klaff@math.utexas.edu, shalen@math.uic.edu

Received: 12 November 2005

Algebraic 63 Geometric Topology, Volume 6 (2006) 